RUNNING HEAD: ADHD and Substance Use Disorder Complexity

\title{
Comorbid Attention Deficit Hyperactivity Disorder and Substance Use Disorder Complexity and Chronicity in Treatment-Seeking Adults
}

Jesse Tyler Young ${ }^{1,3,6}$, MPH (Adjunct Research Associate); Susan Carruthers ${ }^{1}, \mathrm{PhD}$ (Research Fellow); Sharlene Kaye ${ }^{2}, \mathrm{PhD}$ (Research Fellow); Steve Allsop ${ }^{1}, \mathrm{PhD}$ (Professor); Joanne Gilsenan ${ }^{2}$, MPsych(Clin) (Senior Research Officer); Louisa Degenhardt ${ }^{2,6}, \mathrm{PhD}$ (Professor); Geurt van de Glind ${ }^{4,5}$, PhD (Postdoctoral Researcher); Wim van den Brink ${ }^{5}$ PhD (Professor); \& David Preen ${ }^{3}, \mathrm{PhD}$ (Professor)

${ }^{1}$ National Drug Research Institute, Curtin University, Perth, WA, 6008, Australia

${ }^{2}$ National Drug and Alcohol Research Centre, University of New South Wales, Sydney, NSW, 2052, Australia

${ }^{3}$ Centre for Health Services Research, School of Population Health, The University of Western Australia, Perth, WA, 6009, Australia

${ }^{4}$ Trimbos-instituut and ICASA Foundation, Utrecht, 3521, The Netherlands

${ }^{5}$ Amsterdam Institute for Addiction Research, Department of Psychiatry, Academic Medical Center, University of Amsterdam, Amsterdam, The Netherlands

${ }^{6}$ Melbourne School of Population and Global Health, University of Melbourne, Parkville, VIC, 3010, Australia

Word count (Introduction - Conclusion): 3499

Word count (Abstract): 250

Corresponding Author and Institution of the Work:

Mr Jesse Tyler Young

National Drug Research Institute

GPO Box U1987, Perth, WA, 6845, Australia

Tel: +61 864881317

Fax: +61 864881188

Email: jesse.young@curtin.edu.au

Note: This is the accepted version of the following article: Young J T., Carruthers S., Kaye S., Allsop S., Gilsenan J., Degenhardt L., van de Glind G., van den Brink W., Preen D. Comorbid Attention Deficit Hyperactivity Disorder (ADHD) and Substance Use Disorder (SUD) Complexity and Chronicity in Treatment-Seeking Adults. Drug and Alcohol Review. 2015; Epub ahead of print., which has been published in final form at DOI:10.1111/dar.12249 
RUNNING HEAD: ADHD and Substance Use Disorder Complexity

ABSTRACT

\section{Introduction and Aims}

Attention Deficit Hyperactivity Disorder (ADHD) is a known risk factor for substance use disorder (SUD), however the potential additive contribution of comorbid ADHD to drugspecific dependence in SUD populations is largely unknown. The current study aimed to assess this association between ADHD symptoms and drug-specific SUD complexity and chronicity.

\section{Design and Methods}

A cross-sectional survey was administered to a convenience sample of 489 adults receiving SUD treatment at 16 Australian drug and alcohol treatment centres between September 2010 and August 2011. Participants were screened for adult ADHD symptoms using the Adult ADHD Self-report Scale (ASRS). Associations between ADHD screening status and drug-specific SUD complexity and chronicity were assessed using multivariate logistic and modified Poisson regression analysis, controlling for a range of potential confounders.

\section{Results}

Overall, 215 (44.0\%) patients screened positive for concurrent adult ADHD and SUD. After Simes' correction, a significant positive association was observed between ADHD screening status and current amphetamine SUD (OR=1.85; 95\%Cl:1.19-2.36). Patients who screened positive for ADHD were significantly more likely to report SUD history for heavy alcohol use $(\mathrm{OR}=2.05 ; 95 \% \mathrm{Cl}: 1.21-3.45)$ and amphetamine (OR=1.96; 95\% $\mathrm{Cl}: 1.26-3.06)$ as well as significantly increased risk of moderate (3-4 years) duration for benzodiazepines and amphetamine SUDs and long ( $\geq 5$ years) duration for alcohol, opiates other than heroin or methadone, and amphetamine SUDs.

\section{Discussion and Conclusions}


RUNNING HEAD: ADHD and Substance Use Disorder Complexity

The findings provide evidence there is increased drug dependence complexity and chronicity in treatment-seeking SUD patients who screen positively for ADHD, specifically for amphetamine, alcohol, opiates other than heroin or methadone, and benzodiazepines.

\section{Key Words}

ADHD; Substance Use Disorder; ASRS; Comorbidity; Attention Deficit Hyperactivity Disorder; Dependence 
RUNNING HEAD: ADHD and Substance Use Disorder Complexity

\section{Comorbid Attention Deficit Hyperactivity Disorder and Substance Use Disorder Complexity and Chronicity in Treatment-Seeking Adults}

\section{INTRODUCTION:}

Global prevalence of childhood Attention Deficit Hyperactivity Disorder (ADHD) is reported to be between $5-10 \%$ [1-3], with two-thirds of all childhood ADHD cases persisting into adulthood[4,5]. Furthermore, adult ADHD is overrepresented in substance use disorder (SUD) populations and, subject to the sampling methodology applied, prevalence estimates range from $14-44 \%$ [6-10], which is considerably higher than the $3-7 \%$ observed in the community[11-14].

ADHD is an important risk factor for SUDs[15,16]. Research measuring adult ADHD symptoms has demonstrated that people with ADHD are at a four-fold greater risk of SUD than people without ADHD, which is exacerbated if ADHD is not managed effectively[6,12,17]. In some studies, these associations remain after controlling for comorbid conduct disorder (CD) effects[15,18,19].

To date, most studies in this area have compared the association between ADHD and the presence of SUD in general. Comorbid ADHD and SUD has been observed to exacerbate a number of maladaptive SUD outcomes such as earlier drug use initiation, more severe SUD, increased psychiatric comorbidities, hospitalisations, suicide attempts, and HIV-risk behaviours[18,20,21]; thus making the treatment and management of SUD in clinical settings more challenging and less effective[22-24]. The potential effects of concomitant adult ADHD according to drug-specific SUD complexity and chronicity remain unclear, however these specific factors are potentially predictive of poor treatment adherence, treatment setting effectiveness, slower SUD remission, and greater risk of SUD relapse $[21,22,24,25]$ making their examination important for treatment optimisation in these comorbid patients[26]. The few studies in this area have almost exclusively focussed on 
RUNNING HEAD: ADHD and Substance Use Disorder Complexity

small (often $\mathrm{n}<100$ ) patient samples and have not undertaken drug-specific sub-group analyses, or controlled for other potential modifying risk factors[27].

The aims of this study were to: i) estimate the prevalence of adult ADHD symptoms in Australian SUD populations; ii) assess the association between ADHD symptoms and current SUD for different drug types and for polydrug dependence; and iii) explore the effect of concurrent adult ADHD symptoms on the chronicity of SUD in treatment-seeking SUD patients.

\section{METHODS:}

This study used data from the Australian sites of the International ADHD in Substance use disorders Prevalence (IASP) study; the patient characteristics and design of which is described in further detail elsewhere[28].

\subsection{Study Design}

A cross-sectional survey was administered to a convenience sample of adults ( $\geq 18 y e a r s)$ receiving treatment for SUD in Western Australian (WA) and New South Wales (NSW) metropolitan drug and alcohol treatment centres between $1^{\text {st }}$ September, 2010 and $31^{\text {st }}$ August, 2011.

\subsection{Study Sample}

The sample comprised 489 adults attending drug and alcohol treatment centres for clinicallydiagnosed SUD across two Australian States (187 participants from three residential and five outpatient centres in WA and 302 from four residential and four outpatient centres in NSW). Outpatients $(7 \% ; n=34)$ were receiving either methadone maintenance or psychosocial treatments. Inclusion was restricted to adult participants ( $\geq 18$ years) receiving active clinical SUD treatment whose current treatment episode began less than six months ( $\leq 180$ days) 
RUNNING HEAD: ADHD and Substance Use Disorder Complexity

before the administration of the survey. A small fraction of individuals (1.4\%) that initiated treatment prior to six months, with a reported SUD relapse requiring active treatment within six months of the survey were also included. This inclusion criterion was employed to coincide with the six-month Adult ADHD Self-Report Scale (ASRS) symptom assessment period [29], documented as the most reliable period of self-reported ADHD symptoms [30] and to confirm that no patient had achieved the Diagnostic and Statistical Manual of Mental Disorders, Fourth Edition, Text Revision (DSM-IV-R) and the DSM-5 analogous "full SUD remission" and the specified "in sustained remission" criteria [31,32], therefore retaining their comorbid status. All participants were volunteers and were reimbursed AUD\$20 for participation.

\subsection{Assessments}

The study questionnaire was developed by the IASP Study [28] and assessed patient sociodemographics, drug use history, current adult ADHD screening status, ADHD symptom onset, and reported history of ADHD clinical diagnosis. Patient drug-specific use was assessed through self-report of age of onset, current clinically diagnosed drug dependence requiring treatment, and previous total years of problematic regular use.

Patients were screened for adult ADHD symptoms using the validated ASRS six-item (ASRS-6) dichotomous screener scoring method of Kessler et al. [29] which requires endorsement of four out of six current ADHD symptoms and has been previously validated in SUD populations[33-35]. ASRS-6 screening was conducted a median of 18 days (interquartile range $(\mathrm{IQR})=7-36$ ) after SUD treatment commencement in accordance with optimal ADHD screening periods between 14-28 days of abstinence in SUD adults[36-38], reducing the possibility of false-positives due to intoxication or withdrawal symptom overlap. Participants were asked to report the age of impairment onset due to ADHD symptoms. Previous clinical ADHD diagnoses in childhood and/or as an adult were assessed through participant self-report. 
RUNNING HEAD: ADHD and Substance Use Disorder Complexity

The probable presence or absence of concurrent adult ADHD in SUD patients, herein referred to as ADHD-Screened Positive (ADHD-SP) and ADHD-Screened Negative (ADHD$\mathrm{SN}$ ), was identified using the ASRS- 6 with an age of onset criteria (AOC) threshold of $<12$ years, an empirically supported threshold in accordance with the DSM-5 adult ADHD diagnosis requirements[32]. Previous investigation has shown that 95\% of ADHD and only less than $10 \%$ of mood disorders onset prior to 12 years [39]; therefore this AOC threshold was set to minimise potential misclassification bias due to shared symptom presentation between ADHD and mood disorders, documented to create an inflated risk of false positives from increased comorbid prevalence of these disorders in SUD populations[33,40]. Additionally, this AOC threshold aids in differentiating between shared ADHD and SUD symptoms [38], increasing confidence that the reported ADHD symptoms were antecedent to adolescence, where the vast majority of SUDs and mood disorders onset is observed[39,40].

ADHD symptom status was determined according to: i) a positive screening for current symptoms on the ASRS-6 diagnostic tool or; ii) a self-reported ADHD diagnosis as an adult; or iii) a self-reported ADHD diagnosis in childhood accompanied by a positive screening on the ASRS-6 or a self-reported adult ADHD diagnosis. In all cases, positive ADHD symptom status required an $\mathrm{AOC}<12$ years.

Participants with adult-onset ( $\geq 18$ years) SUD who reported a childhood ADHD diagnosis but did not screen positive on the ASRS- 6 were excluded $(n=1)$ from the ADHD-SP group due to the possibility of ADHD remission as an adult[31,41], thus lacking evidence for concurrent SUD and ADHD.

Self-reported current SUDs requiring clinical treatment were analysed as binary outcomes. Drugs investigated included: alcohol, nicotine, heroin, methadone, other opiates (i.e. morphine, oxycodone, buprenorphine), benzodiazepines, cocaine, amphetamine, ecstasy, and cannabis. Use of prescribed (non-diverted) pharmaceutical drugs, such as methadone 
RUNNING HEAD: ADHD and Substance Use Disorder Complexity

maintenance treatment, was excluded from analysis ensuring that only illicit drug use and unsanctioned or pharmaceutical drug misuse was evaluated. Poly-drug dependence was assessed by creating an index score indicating the aggregate number of current SUDS requiring treatment reported by each participant.

One year of regular drug use was defined as two consecutive days of use or at least three days of use per week for a period of six months or more with a self-reported 'problematic' use indication as a proxy measure for the DSM-IV-R and DSM-5 criteria for SUD[31,32]. Adhering to the DSM-IV-R sustained full SUD remission criteria, periods of regular use were summed into a total number of years of use, excluding periods of abstinence exceeding 12 months[31]. The total years of problematic regular drug use was collapsed into a binary variable indicating the presence/absence of previous dependent use (i.e. no years versus one or more years of regular use), and assessed separately for each drug. Increased SUD complexity was defined as experiencing a greater number of current SUDs requiring treatment and more prior SUDs.

Additional examination was conducted on the duration of dependent use for drugs that exhibited a primary significant binary association between ADHD and dependent use as a binary variable. For these variables, four ordinal categories of SUD duration were created: no dependent use (reference condition), short (1-2 years), moderate (3-4 years), and long duration ( $\geq 5$ years) of dependent use.

\subsection{Statistical Methods}

All analyses were conducted using SPSS Version 20.0. All primary tests were two-tailed with significance set at $p<0.05$. Additionally, Simes' correction $\left(P_{\text {Simes }}: p<0.01\right)$ was applied to adjust the p-value for multiple testing on all primary tests (i.e. Tables 2-5); Simes' method has been asserted to control for type I error while avoiding the potential for overcorrection affiliated with the Bonferroni method[42,43]. 
RUNNING HEAD: ADHD and Substance Use Disorder Complexity

Descriptive statistics were calculated for all variables. Crude (unadjusted) comparative analyses (independent samples t-tests and chi-square) were initially performed comparing continuous and categorical outcomes respectively, between ADHD-SP and ADHD-SN groups. Crude ORs $(95 \% \mathrm{Cls})$ were calculated by univariate logistic regression for all binary outcome variables.

The association between concomitant ADHD symptom status (ADHD-SP versus ADHD-SN) and SUD complexity and chronicity was estimated separately for each drug type while controlling for a range of sociodemographic, clinical, and behavioural characteristics. Multivariate logistic regression modelling was used to determine the association between ADHD and drug-specific current SUD, SUD history, and SUD comorbidity index. For significant associations from collapsed binary SUD history variables, additional modified loglinked Poisson regression models utilising robust sandwich variance were fitted to uncorrelated ordinal factor variables for SUD duration, predicting binary ADHD screening status as outlined previously by Zou (2004)[44].

All regression models adjusted for age, gender, ethnicity, education level, marital status, history of traumatic brain injury, living arrangements, and offending history. Covariates were selected due to demonstrated or likely influence on the association between ADHD and SUD $[18,45]$. Originally, a smaller nested-model including age, gender, ethnicity and education level was fit, however the larger model was a more parsimonious fit [46] for the outcomes assessed. It was planned to adjust for self-reported CD and ODD diagnoses, as both variables represent common covariates in comorbid ADHD and SUD research[47-49]. However, these factors were not included in the regression models due to low study sample prevalence $(0.8 \%$ and $0.4 \%$, respectively).

This study received approval from the Curtin University and the University of New South Wales Human Research Ethics Committees. 
RUNNING HEAD: ADHD and Substance Use Disorder Complexity

\section{RESULTS:}

Characteristics of study participants overall and by ADHD subgroup are displayed in Table 1. Overall, 215 (44.0\%) participants screened positive for concurrent adult ADHD symptom status and SUD. Most of the sample comprised inpatients from residential treatment centres (93.1\%;n=455), however ADHD screening status was not associated with treatment modality $\left(x^{2}=0.487 ; p=0.485\right)$. The ADHD-SP group was significantly younger than the ADHD-SN group $(t=3.865 ; p<0.001)$. No other significant differences between ADHD screening status groups were observed (Table 1).

$<$ Table 1>

\subsection{ADHD and current SUDs}

A summary of the reported current SUDs at the time of questionnaire completion is presented in Table 2. Primary multivariate logistic regression adjusting for potential confounders indicated an increased odds for the ADHD-SP group compared to the ADHDSN group of having SUDs for amphetamine and cannabis (Table 2). After Simes' correction $\left(P_{\text {Simes }}: p<0.01\right)$ was applied, only the association for amphetamine SUD remained significant $(p=0.007)$.

In total, $77.7 \%(n=380)$ of the sample reported multiple current SUDs at the time of the study, with $26.4 \%(n=129)$ reporting four or more SUDs concurrently. Primary multivariate logistic regression modelling revealed that, compared to the ADHD-SN group, the ADHD-SP group was significantly more likely to have four or more concurrent SUDs (OR=2.06; 95\%Cl:1.03-4.11). However, after Simes' correction, this association did not remain significant $(p=0.042)$.

\subsection{Prior SUD History Reported}

Prior SUD history for each drug is summarised in Table 2. A total of 476 participants (97.3\%) reported multiple prior SUDs, with the median number of SUDs being 5 (range 1-11). 
RUNNING HEAD: ADHD and Substance Use Disorder Complexity

Primary multivariate logistic regression indicated significant positive associations between ADHD screening status and prior SUD history for heavy alcohol use, amphetamine, opiates other than heroin or methadone, benzodiazepines, and ecstasy (Table 2). After Simes' correction, only the association for heavy alcohol use $(p=0.007)$ and amphetamine $(p=0.003)$ SUD history remained significant.

$<$ Table 2>

\subsection{ADHD Status and SUD Duration}

The estimated prevalence risk ratios (PRR) from modified Poisson regression analysis describing the association between ADHD screening status and SUD duration are presented in Table 3. Primary analyses indicated that ADHD-SP patients were significantly more likely to report short (i.e. 1-2 years) durations of SUD for ecstasy as well as short and long (i.e. $\geq 5$ years) durations of SUD for opiates other than heroin or methadone. In addition, the ADHDSP group was significantly more likely to report moderate (i.e. 3-4 years) and long durations of benzodiazepines SUD as well as short, moderate, and long durations of amphetamine SUD. The ADHD-SP group was also significantly more likely to report long durations of SUD for heavy alcohol use (Table 3). After Simes' correction, only the risk for moderate durations of benzodiazepines and amphetamine SUDs as well as long durations for opiates other than heroin or methadone, heavy alcohol use, and amphetamine SUDs remained significant.

$<$ Table 3>

\section{DISCUSSION:}

\subsection{Prevalence of probable adult ADHD in SUD patients}

Just under half (44.0\%) of the adult patients with SUD in the current study screened positive for current ADHD symptoms present prior to 12 years of age, representing a seven-fold increase compared to a recent ASRS screening prevalence estimate in an Australian representative community sample (6.2\%)[11]. The screening prevalence estimate observed 
RUNNING HEAD: ADHD and Substance Use Disorder Complexity

in our sample was at the upper range limit when compared to other published studies using the ASRS in SUD populations (range 8-44\%)[7-10,33]. This large prevalence variability is possibly due to a variety of potential influences such as cross-national variation in screening methodology, SUD treatment availability, or divergent clinical SUD characteristics and drugspecific SUD distribution of the patients seeking treatment. It is not possible to identify the main influences using the current methodology. However, as these comparative research studies utilised no AOC threshold, our AOC of 12 years suggests that the high ADHD screening prevalence observed in our study is not due to less conservative ADHD screening methodology. The ASRS-6 has been shown to have good sensitivity (84-88\%) in identifying ADHD in treatment-seeking SUD patients, however ASRS-6 screening alone resulted in a small amount of false positives and slightly inflated prevalence estimates when compared to a 'gold-standard' clinical diagnostic interview in the IASP study[35]. Therefore, although accurate in the majority of cases, the ARSR-6 ADHD status in the current study indicates the presence of ADHD symptoms and should not be interpreted as a DSM-IV or DSM-5 diagnostic indication of adult ADHD prevalence in treatment-seeking SUD patients.

\subsection{ADHD screening status and SUD complexity and chronicity}

In this population of treatment-seeking SUD patients, compared to the ADHD-SN group, the ADHD-SP group was approximately two times more likely to have a current amphetamine SUD and a reported history of amphetamine and alcohol SUDs. Other studies have observed similar positive associations between ADHD and SUD in general $[15,50]$, as well as for other amphetamine-type psychostimulant SUDs [45,51,52], cocaine SUD [8,33], cannabis SUD [17], and alcohol SUD [15,50,51] in particular. Previous investigations have reported that ADHD status is positively associated with increased frequency of the use of cocaine, other illicit psychostimulants [52], benzodiazepines, and opiates [48] compared to controls. It is possible that neurobiological differences, impaired executive functioning, and elevated impulsivity contribute to increased SUD complexity and chronicity in individuals with ADHD compared to normal controls[53-57]. Although it is likely that comorbid ADHD 
RUNNING HEAD: ADHD and Substance Use Disorder Complexity

increases vulnerability to drug and polydrug dependence in general, the drug-specific pattern of SUD increase in treatment-seeking SUD/ADHD-SP above what is seen in SUD/ADHD-SN patients in our study indicates this association is particularly evident with amphetamine.

Although previous studies report associations between ADHD and SUDs for other psychostimulants (e.g., cocaine)[8,33], it is unclear why concurrent ADHD symptoms were associated with increased amphetamine and not cocaine and ecstasy SUD prevalence. However, these psychostimulant drugs act differentially on three primary neurotransmitter systems, eliciting discrete patterns of dopamine, norepinephrine, and serotonin transporter reuptake inhibition[58,59]. These neurotransmitter receptors may function, be represented differently, or be differentially available in individuals with ADHD and thus, distinct neuropsychopharmacological interactions likely contribute to psychostimulant-specific behavioural reinforcement effects in these individuals[60,61]. Furthermore, contrasting drugspecific modes of administration, duration of effect and pharmacokinetic properties (e.g., bioavailability, half-life) of these psychostimulants possibly interact with neurobiologicallyderived behavioural features associated with ADHD-related executive function deficits such as increased reward threshold, (i.e., requiring more immediacy, frequency and salience of reinforcers), impulse control and sensitisation induction[62]. However, the contribution of these factors to divergent psychostimulant dependence patterns cannot be ascertained with the current methodology and represent areas for future examination.

For drug-specific positive associations between ADHD screening status and reported history of SUD (i.e. amphetamine, alcohol, opiates other than heroin and methadone, and benzodiazepines), significant prevalence risk estimates attributable to ADHD symptom status occurred most frequently when SUD duration was $\geq 5$ years. Although the association between ADHD screening status and discrete SUD duration levels has not been previously explored, the current findings corroborate previous assertions that comorbid ADHD results in longer SUD durations, i.e. a more chronic course of SUD[24]. Additionally, neuroimaging studies have identified biological links between ADHD and both SUD-craving brain activation 
RUNNING HEAD: ADHD and Substance Use Disorder Complexity

and SUD-impulsivity [53], known risk factors for SUD persistence and the susceptibility to relapse[24,54]. These findings imply that the identification of comorbid ADHD in SUD treatment settings may provide early indication of symptoms that may predict a greater risk of delayed SUD remission and SUD relapse. This may present clinicians with an opportunity to efficiently target, integrate, and implement treatment strategies addressing both conditions concurrently, shown to be more effective compared to parallel or segmented comorbid treatment approaches[26,63]. Interestingly, the observed ADHD symptom-related increase in alcohol and benzodiazepine SUD duration raises the possibility that impaired executive functioning and behavioural inhibition may have particular relevance for these drug-specific SUD patterns. Prior research has postulated that reduced forebrain dopamine levels share a role in both ADHD-related executive deficits and alcohol SUD development[64]. Benzodiazepines have been shown to alter reward threshold and behavioural inhibition[65], both of which are associated with ADHD-related executive function deficits.

Our results indicate that SUD patients with comorbid ADHD symptoms report a pattern of increased SUD complexity and chronicity (i.e. experiencing more current SUDs, prior SUDs, and longer dependency) for amphetamine, alcohol, opiates other than heroin or methadone, and benzodiazepine drugs than ADHD-SN individuals with SUD. The ability to identify ADHD in SUD patients at treatment initiation may be advantageous in determining SUD patient characteristics that may place them at increased risk for poorer treatment outcomes. Previous research has indicated that comorbid ADHD is a risk factor for extended SUD duration, slower remission rates, and a higher prevalence of substance-induced disorders, further complicating the treatment and management of SUD[22-24]. Given their accuracy, ease of administration, and time-effectiveness, screening tools such as the ASRS-6 may be especially efficacious for the detection of comorbid ADHD in drug treatment centre settings. Therefore, not only could this assist the tailoring of SUD patient treatments but it may also enable additional integrated treatments of adult ADHD and SUD, representing a more holistic approach[26]. 
RUNNING HEAD: ADHD and Substance Use Disorder Complexity

To our knowledge this study represents the largest assessment of the association between comorbid adult ADHD symptom status and drug-specific SUD complexity and chronicity while controlling for multiple confounders. Additionally, the ADHD-SP and drug-specific SUD associations discussed here remained significant after the Simes' correction was applied, further substantiating these inferences. However, some study limitations exist. The current study relied on participant self-reports which may impact on the data obtained; social acceptability, perceived consequences of disclosure, question comprehension, long-term recall duration and drug-related memory deficits are known factors influencing the accuracy of drug use self-reporting. Self-reported data, however, have been found to be sufficiently reliable and valid to inform about drug use patterns and associated problems $[66,67]$ and in the assessment of childhood and current symptoms of ADHD among adults[68]. The measure of SUD chronicity was derived from a proxy of self-reported 'problematic' regular drug use, however this measure is limited as it does not fully assess the drug dependence threshold for clinical impairment (i.e. withdrawal, increased tolerance, and impaired control). Previous clinical diagnoses of $C D$ and ODD were only assessed through binary self-report and as these diagnoses receive limited public attention in Australia, the recall of these diagnoses by participants is likely biased, resulting in the unusually low prevalence observed in the study group. Conversely, the possibility of patient-motivated ADHD diagnosis overreporting cannot be excluded. Additionally, as the study design was a cross-sectional survey, the temporal sequence of morbidity is not known. The fraction our sample represents of the total patients in the recruitment sites during the sampling period is also undetermined. However, the current sample characteristics concord with Australian drug and alcohol treatment patients in regards to age, socioeconomic status, and drug requiring treatment[69]. Finally, while a validated and widely-used tool in the literature, the ASRS-6 indicates ADHD symptom presence and does not definitively indicate ADHD diagnosis to the same extent as a clinical psychiatric examination. 
RUNNING HEAD: ADHD and Substance Use Disorder Complexity

\section{CONCLUSION:}

The findings of this study indicate that adult ADHD is highly overrepresented in treatmentseeking SUD populations as compared with their community counterparts in Australia. Additionally, comorbid adult ADHD symptoms are associated with elevated drug dependence complexity and chronicity in treatment-seeking SUD populations, specifically for amphetamine, alcohol, opiates other than heroin and methadone, and benzodiazepines. The fact that this ADHD-related SUD pattern was observed in the current sample, where all participants had a severe current SUD necessitating treatment, is indicative of a robust association.

\section{AUTHOR DISCLOSURES:}

\section{Role of Funding Source}

This research was funded by a Curtin University Strategic Research Faculty Grant. However, the funding source had no additional role in the research methodology; the collection, analysis, or interpretation of data; the writing of the manuscript; or in the decision to submit the article for publication.

\section{Contributors}

Authors van de Glind and van den Brink wrote the original IASP research proposal and developed the research design and protocol. Authors Kaye, Degenhardt, Allsop, and Carruthers further adapted and modified the research design and protocol, and coordinated the Australian arm of the study. Authors Kaye, Carruthers, Young, and Gilsenan administered participant interviews and were responsible for data entry, quality control auditing, and data management. Authors Young and Preen conducted the data analysis. Author Young wrote the initial draft manuscript. All authors contributed comments to the interpretation and synthesis of results, and approved the final manuscript submitted.

\section{Conflict of Interest}


RUNNING HEAD: ADHD and Substance Use Disorder Complexity

For coordination of the IASP study, (see also Van de Glind et al., 2013c) grants were received from pharmaceutical companies (Shire, Eli Lilly \& company, Janssen Cilag), from participating institutes and from three not for profit organizations: the Waterloo Foundation, the Noaber Foundation and the Augeo Foundation.

The funding companies, institutes and foundations did not have and will not have influence on any aspect of the study, including research questions, data sampling, data management, data analyses and publishing results. Since September 2010 the IASP study and the ICASA Foundation function independent from pharmaceutical companies.

G. Van de Glind was on one occasion consultant for Shire, for which he refused payment. In 2013 he received an unrestricted travel grant from Neurotech and he is (unpaid) member of the advisory board of Neurotech.

W. van den Brink has received a fee from Eli Lilly for organizing a symposium on the role of impulsivity in psychiatric disorders and a speaker's fee from Eli Lilly for a presentation on the relationship between ADHD and addiction.

Apart from the funding resources disclosed in the acknowledgements section, the above mentioned authors and all other authors declare no conflicts of interest.

\section{Acknowledgements}

This research was funded by a Curtin University Strategic Research Faculty Grant. The authors would like to acknowledge the contributions by the treatment agencies participating in this research. Louisa Degenhardt is supported by an NHMRC Principal Research Fellowship. The National Drug Research Institute at Curtin University and The National Drug and Alcohol Research Centre at the University of NSW are supported by funding from the 
RUNNING HEAD: ADHD and Substance Use Disorder Complexity

Australian Government under the Substance Misuse Prevention and Service Improvements Grants Fund. 
RUNNING HEAD: ADHD and Substance Use Disorder Complexity

\section{REFERENCES:}

1. Skounti, M., Philalithis, A. \& Galanakis, E. (2007) Variations in prevalence of attention deficit hyperactivity disorder worldwide, Eur J Pediatr, 166, 117-123. doi:10.1007/s00431-006-0299-5

2. Polanczyk, G., De Lima, M. S., Horta, B. L., Biederman, J. \& Rohde, L. A. (2007) The Worldwide Prevalence of ADHD: A Systematic Review and Metaregression Analysis, Am J Psychiatry, 164, 942-948. doi:10.1176/appi.ajp.164.6.942

3. Swanson, J. M., Sergeant, J. A., Taylor, E. et al. (1998) Attention-deficit hyperactivity disorder and hyperkinetic disorder, Lancet, 351, 429-433. doi:10.1016/s01406736(97)11450-7

4. Sullivan, M. A. \& Levin, F. R. (2001) Attention Deficit/Hyperactivity Disorder and Substance Abuse, Ann N Y Acad Sci, 931, 251-270. doi:10.1111/j.17496632.2001.tb05783.x

5. Wender, P. H., Wolf, L. E. \& Wasserstein, J. (2001) Adults with ADHD. An overview., Ann N Y Acad Sci, 931, 1-16. doi:10.1111/j.1749-6632.2001.tb05770.x

6. Schubiner, H., Tzelepis, A., Milberger, S. et al. (2000) Prevalence of AttentionDeficit/Hyperactivity Disorder and Conduct Disorder Among Substance Abusers, J Clin Psychiatry, 61, 244-251. doi:10.4088/JCP.v61n0402

7. Mcaweeney, M., Rogers, N. L., Huddleston, C., Moore, D. \& Gentile, J. P. (2010) Symptom Prevalence of ADHD in a Community Residential Substance Abuse Treatment Program, J Atten Disord, 13, 601-608. doi:10.1177/1087054708329973

8. Pérez De Los Cobos, J., Siñol, N., Puerta, C. et al. (2011) Features and prevalence of patients with probable adult attention deficit hyperactivity disorder who request treatment for cocaine use disorders, Psychiatry Res, 185, 205-210.

doi:10.1016/j.psychres.2009.03.019

9. Van Emmerik-Van Oortmerssen, K., Van De Glind, G., Van Den Brink, W. et al. (2012) Prevalence of attention-deficit hyperactivity disorder in substance use disorder patients: A meta-analysis and meta-regression analysis, Drug Alcohol Depend, 122, 11-19. doi:10.1016/j.drugalcdep.2011.12.007

10. Van De Glind, G., Konstenius, M., Koeter, M. W. J. et al. (2013) Variability in the prevalence of adult ADHD in treatment seeking substance use disorder patients: results from an international multi-center study exploring DSM-IV and DSM-5 criteria, Drug Alcohol Depend, In Press. doi:10.1016/j.drugalcdep.2013.09.026

11. Das, D., Cherbuin, N., Butterworth, P., Anstey, K. J. \& Easteal, S. (2012) A Population-Based Study of Attention Deficit/Hyperactivity Disorder Symptoms and 
RUNNING HEAD: ADHD and Substance Use Disorder Complexity

Associated Impairment in Middle-Aged Adults, PLOS ONE, 7, e31500.

doi:10.1371/journal.pone.0031500

12. Fayyad, J., De Graaf, R., Kessler, R. et al. (2007) Cross-national prevalence and correlates of adult attention-deficit hyperactivity disorder, Br J Psychiatry, 190, 402-409. doi:10.1192/bjp.bp.106.034389

13. Kessler, R. C., Adler, L., Barkley, R. et al. (2006) The prevalence and correlates of adult ADHD in the United States: results from the National Comorbidity Survey Replication, Am J Psychiatry, 163, 716-723. doi:10.1176/appi.ajp.163.4.716

14. Polanczyk, G., Laranjeira, R., Zaleski, M. et al. (2010) ADHD in a representative sample of the Brazilian population: estimated prevalence and comparative adequacy of criteria between adolescents and adults according to the item response theory, Int $\mathrm{J}$ Methods Psychiatr Res, 19, 177-184. doi:10.1002/mpr.319

15. Wilens, T. E., Martelon, M., Joshi, G. et al. (2011) Does ADHD predict substance-use disorders? A 10-year follow-up study of young adults with ADHD, J Am Acad Child Adolesc Psychiatry, 50, 543-53. doi:10.1016/j.jaac.2011.01.021

16. Lee, S. S., Humphreys, K. L., Flory, K., Liu, R. \& Glass, K. (2011) Prospective association of childhood attention-deficit/hyperactivity disorder (ADHD) and substance use and abuse/dependence: A meta-analytic review, Clin Psychol Rev, 31, 328-341.

doi:10.1016/j.cpr.2011.01.006

17. Levin, F. R., Evans, S. M. \& Kleber, H. D. (1998) Prevalence of adult attention-deficit hyperactivity disorder among cocaine abusers seeking treatment, Drug Alcohol Depend, 52, 15-25. doi:10.1016/s0376-8716(98)00049-0

18. Arias, A. J., Gelernter, J., Chan, G. et al. (2008) Correlates of co-occurring ADHD in drug-dependent subjects: Prevalence and features of substance dependence and psychiatric disorders, Addict Behav, 33, 1199-1207. doi:10.1016/j.addbeh.2008.05.003

19. Elkins, I. J., Mcgue, M. \& lacono, W. G. (2007) Prospective effects of attentiondeficit/hyperactivity disorder, conduct disorder, and sex on adolescent substance use and abuse, Arch Gen Psychiatry, 64, 1145-1152. doi:10.1001/archpsyc.64.10.1145

20. Abrantes, A. M., Strong, D. R., Ramsey, S. E., Kazura, A. N. \& Brown, R. A. (2006) HIV-Risk Behaviors Among Psychiatrically Hospitalized Adolescents With And Without Comorbid SUD, J Dual Diagn, 2, 85-100. doi:10.1300/J374v02n03_08

21. Tamm, L., Trello-Rishel, K., Riggs, P. et al. (2013) Predictors of treatment response in adolescents with comorbid substance use disorder and attention-deficit/hyperactivity disorder, J Subst Abuse Treat, 44, 224-230. doi:10.1016/j.jsat.2012.07.001 
RUNNING HEAD: ADHD and Substance Use Disorder Complexity

22. Carroll, K. M. \& Rounsaville, B. J. (1993) History and significance of childhood attention deficit disorder in treatment-seeking cocaine abusers, Compr Psychiatry, 34, 75-82. doi:10.1016/0010-440x(93)90050-e

23. Tang, Y.-L., Kranzler, H. R., Gelernter, J., Farrer, L. A. \& Cubells, J. F. (2007) Comorbid Psychiatric Diagnoses and Their Association with Cocaine-Induced Psychosis in Cocaine-Dependent Subjects, Am J Addict, 16, 343-351. doi:10.1080/10550490701525723

24. Wilens, T. E., Biederman, J. \& Mick, E. (1998) Does ADHD Affect the Course of Substance Abuse?: Findings From a Sample of Adults With and Without ADHD, Am J Addict, 7, 156-163. doi:10.1111/j.1521-0391.1998.tb00330.x

25. Tiet, Q. Q., Ilgen, M. A., Byrnes, H. F., Harris, A. H. S. \& Finney, J. W. (2007) Treatment setting and baseline substance use severity interact to predict patients' outcomes, Addiction, 102, 432-440. doi:10.1111/j.1360-0443.2006.01717.x

26. Van Emmerik-Van Oortmerssen, K., Vedel, E., Koeter, M. et al. (2013) Investigating the efficacy of integrated cognitive behavioral therapy for adult treatment seeking substance use disorder patients with comorbid ADHD: study protocol of a randomized controlled trial, BMC Psychiatry, 13, 132. doi:10.1186/1471-244X-13-132

27. Yewers, T. M., Hay, D. A. \& Barton, A. (2005) Attention deficit hyperactivity disorder and severity of drug use in a sample of adult male drug users, Australian Psychologist, 40, 109-117. doi:10.1080/00050060500094597

28. Van De Glind, G., Van Emmerik-Van Oortmerssen, K., Carpentier, P. J. et al. (2013) The International ADHD in Substance Use Disorders Prevalence (IASP) study: background, methods and study population, Int J Methods Psychiatr Res, 22, 232-244.

doi:10.1002/mpr.1397

29. Kessler, R. C., Adler , L., Ames, M. et al. (2005) The World Health Organization adult ADHD self-report scale (ASRS): a short screening scale for use in the general population, Psychol Med, 35, 245-256. doi:10.1017/S0033291704002892

30. Fatseas, M., Debrabant, R. \& Auriacombe, M. (2012) The diagnostic accuracy of attention-deficit/hyperactivity disorder in adults with substance use disorders, Curr Opin Psychiatry, 25, 219. doi:10.1097/YCO.0b013e3283523d7c

31. American Psychiatric Association (2000) Diagnostic and Statistical Manual of Mental Disorders, Fourth Edition, Text Revision. (Washington, DC, American Psychiatric Association).

32. American Psychiatric Association (2013) Diagnostic and Statistical Manual of Mental Disorders, Fifth Edition (Arlington, VA, American Psychiatric Association). 
RUNNING HEAD: ADHD and Substance Use Disorder Complexity

33. Daigre, C., Ramos-Quiroga, J., Valero, S. et al. (2009) Adult ADHD Self-Report Scale (ASRS-v1.1) symptom checklist in patients with substance use disorders, Actas Esp Psiquiatr, 37, 299-305.

34. Perez, E. P. \& Garcia, C. P. (2007) ASRS v.1.1., a tool for attentiondeficit/hyperactivity disorder screening in adults treated for addictive behaviors: psychometric properties and estimated prevalence, Adicciones, 19, 393-407.

35. Van De Glind, G., Van Den Brink, W., Koeter, M. W. J. et al. (2013) Validity of the Adult ADHD Self-report Scale (ASRS) as a screener for adult ADHD in treatment seeking substance use disorder patients, Drug Alcohol Depend, 132, 587-596.

doi:10.1016/j.drugalcdep.2013.04.010

36. Milin, R., Loh, E., Chow, J. \& Wilson, A. (1997) Assessment of Symptoms of Attention-Deficit Hyperactivity Disorder in Adults With Substance Use Disorders, Psychiatr Serv, 48, 1378-1380.

37. Wilens, T. E. (2004) Attention-deficit/hyperactivity disorder and the substance use disorders: the nature of the relationship, subtypes at risk, and treatment issues, Psychiatr Clin North Am, 27, 283-301. doi:10.1016/S0193-953X(03)00113-8

38. Schubiner, H. (2005) Substance Abuse in Patients with Attention-Deficit Hyperactivity Disorder: Therapeutic Implications, CNS Drugs, 19, 643-655.

39. Kessler, R. C., Berglund, P., Demler, O. et al. (2005) Llfetime prevalence and age-ofonset distributions of dsm-iv disorders in the national comorbidity survey replication, Arch Gen Psychiatry, 62, 593-602. doi:10.1001/archpsyc.62.6.593

40. Kessler, R. C. (2004) The epidemiology of dual diagnosis, Biol Psychiatry, 56, 730737. doi:10.1016/j.biopsych.2004.06.034

41. Faraone, S. V., Biederman, J. \& Mick, E. (2006) The age-dependent decline of attention deficit hyperactivity disorder: a meta-analysis of follow-up studies, Psychol Med, 36, 159-165. doi:10.1017/S003329170500471X

42. Simes, R. J. (1986) An Improved Bonferroni Procedure for Multiple Tests of Significance, Biometrika, 73, 751-754. doi:10.2307/2336545

43. Rødland, E. A. (2006) Simes' Procedure Is 'Valid on Average', Biometrika, 93, 742746. doi:10.2307/20441320

44. Zou, G. (2004) A Modified Poisson Regression Approach to Prospective Studies with Binary Data, Am J Epidemiol, 159, 702-706. doi:10.1093/aje/kwh090 
RUNNING HEAD: ADHD and Substance Use Disorder Complexity

45. Rosler, M., Retz, W., Yaqoobi, K., Burg, E. \& Retz-Junginger, P. (2009) Attention deficit/hyperactivity disorder in female offenders: prevalence, psychiatric comorbidity and psychosocial implications, Eur Arch Psychiatry Clin Neurosci, 259, 98-105. doi:10.1007/s00406-008-0841-8

46. Bentler, P. M. \& Bonett, D. G. (1980) Significance Tests and Goodness of Fit in the Analysis of Covariance Structures, Psychol Bull, 88, 588-606.

47. Reimherr, F. W., Marchant, B. K., Olsen, J. L., Wender, P. H. \& Robison, R. J. (2011) Oppositional Defiant Disorder in Adults With ADHD, J Atten Disord, 1-12. doi:10.1177/1087054711425774

48. Carpentier, P. J., Van Gogh, M. T., Knapen, L. J. M., Buitelaar, J. K. \& De Jong, C. a. J. (2011) Influence of Attention Deficit Hyperactivity Disorder and Conduct Disorder on Opioid Dependence Severity and Psychiatric Comorbidity in Chronic Methadone-Maintained Patients, Eur Addict Res, 17, 10-20. doi:10.1159/000321259

49. Fischer, M., Barkley, R. A., Smallish, L. \& Fletcher, K. (2002) Young Adult Follow-Up of Hyperactive Children: Self-Reported Psychiatric Disorders, Comorbidity, and the Role of Childhood Conduct Problems and Teen CD, J Abnorm Child Psychol, 30, 463-475. doi:10.1023/a:1019864813776

50. Milberger, S., Biederman, J., Faraone, S. V., Wilens, T. \& Chu, M. P. (1997) Associations Between ADHD and Psychoactive Substance Use Disorders, Am J Addict, 6, 318-329. doi:10.1111/j.1521-0391.1997.tb00413.x

51. Barkley, R. A., Murphy, K. R. \& Fischer, M. (2008) ADHD in Adults: What the Science Says (New York, NY, The Guilford Press).

52. Wilens, T. E., Adamson, J., Sgambati, S. et al. (2007) Do Individuals with ADHD SelfMedicate with Cigarettes and Substances of Abuse? Results from a Controlled Family Study of ADHD, Am J Addict, 16, 14-23. doi:10.1080/10550490601082742

53. Crunelle, C. L., Veltman, D. J., Van Emmerik-Van Oortmerssen, K., Booij, J. \& Van Den Brink, W. (2013) Impulsivity in adult ADHD patients with and without cocaine dependence, Drug Alcohol Depend, 129, 18-24. doi:10.1016/j.drugalcdep.2012.09.006

54. Frodl, T. (2010) Comorbidity of ADHD and Substance Use Disorder (SUD): A Neuroimaging Perspective, J Atten Disord, 14, 109-120. doi:10.1177/1087054710365054

55. Volkow, N. D., Wang, G., Newcorn, J. \& Et Al. (2007) Depressed dopamine activity in caudate and preliminary evidence of limbic involvement in adults with attentiondeficit/hyperactivity disorder, Arch Gen Psychiatry, 64, 932-940. doi:10.1001/archpsyc.64.8.932 
RUNNING HEAD: ADHD and Substance Use Disorder Complexity

56. Wilens, T. E., Martelon, M., Fried, R. et al. (2011) Do Executive Function Deficits Predict Later Substance Use Disorders Among Adolescents and Young Adults?, J Am Acad Child Adolesc Psychiatry, 50, 141-149. doi:10.1016/j.jaac.2010.11.010

57. Brady, K. T. \& Sinha, R. (2005) Co-occurring mental and substance use disorders: the neurobiological effects of chronic stress, Am J Psychiatry, 162, 1483-1493. doi:10.1176/appi.ajp.162.8.1483

58. Rothman, R. B. \& Baumann, M. H. (2003) Monoamine transporters and psychostimulant drugs, Eur J Pharmacol, 479, 23-40. doi:10.1016/j.ejphar.2003.08.054

59. Sulzer, D., Sonders, M. S., Poulsen, N. W. \& Galli, A. (2005) Mechanisms of neurotransmitter release by amphetamines: A review, Prog Neurobiol, 75, 406-433. doi:10.1016/j.pneurobio.2005.04.003

60. Oades, R. D. (2008) Dopamine-serotonin interactions in attention-deficit hyperactivity disorder (ADHD), in: Giuseppe Di Giovann, V. D. M. \& Ennio, E. (Eds.) Prog Brain Res, pp. 543-565. doi:10.1016/S0079-6123(08)00926-6

61. Tripp, G. \& Wickens, J. R. (2009) Neurobiology of ADHD, Neuropharmacology, 57, 579-589. doi:10.1016/j.neuropharm.2009.07.026

62. Solanto, M. V. (1998) Neuropsychopharmacological mechanisms of stimulant drug action in attention-deficit hyperactivity disorder: a review and integration, Behav Brain Res, 94, 127-152. doi:10.1016/S0166-4328(97)00175-7

63. Brunette, M., Mueser, K. \& Drake, R. (2004) A review of research on residential programs for people with severe mental illness and co-occurring substance use disorders, Drug Alcohol Rev, 23, 471-481. doi:10.1080/09595230412331324590

64. Smith, B. H., Molina, B. S. \& Pelham, W. (2002) The clinically meaningful link between alcohol use and attention deficit hyperactivity disorder, Alcohol Res Health, 26, 122-129.

65. Tallman, J. F., Paul, S. M., Skolnick, P. \& Gallager, D. W. (1980) Receptors for the Age of Anxiety: Pharmacology of the Benzodiazepines, Science, 207, 274-281. doi:10.2307/1684170

66. Darke, S. (1998) Self-report among injecting drug users: A review, Drug Alcohol Depend, 51, 253-263. doi:10.1016/S0376-8716(98)00028-3

67. Ledgerwood, D. M., Goldberger, B. A., Risk, N. K., Lewis, C. E. \& Kato Price, R. (2008) Comparison between self-report and hair analysis of illicit drug use in a community sample of middle-aged men, Addict Behav, 33, 1131-1139.

doi:10.1016/j.addbeh.2008.04.009 
RUNNING HEAD: ADHD and Substance Use Disorder Complexity

68. Murphy, P. \& Schachar, R. (2000) Use of Self-Ratings in the Assessment of Symptoms of Attention Deficit Hyperactivity Disorder in Adults, Am J Psychiatry, 157, 11561159. doi:10.1176/appi.ajp.157.7.1156

69. Australian Institute of Health and Welfare (2013) Alcohol and other drug treatment services in Australia 2011-12. Drug treatment series 21 Cat no HSE139. (Canberra, AIHW). 
RUNNING HEAD: ADHD and Substance Use Disorder Complexity

TABLES:

Table 1 - Sociodemographic characteristics of study participants.

\begin{tabular}{|c|c|c|c|c|}
\hline Characteristic & $\begin{array}{r}\text { ADHD-SP } \\
N(\%) \\
215(44.0 \%) \\
\end{array}$ & $\begin{array}{r}\text { ADHD-SN } \\
N(\%) \\
274(56.0 \%) \\
\end{array}$ & $\begin{array}{r}\text { All participants } \\
\mathrm{N}(\%) \\
\mathrm{N}=489 \\
\end{array}$ & $\mathrm{p}$-value \\
\hline $\begin{array}{l}\text { Gender } \\
\text { - Female }\end{array}$ & $98(45.6 \%)$ & $142(51.8 \%)$ & 240 (49.1\%) & $0.170^{b}$ \\
\hline Age (Years \pm SD) & $32.9 \pm 8.5$ & $36.0 \pm 8.8$ & $34.6 \pm 8.8$ & $<0.001^{\mathrm{a}}$ \\
\hline $\begin{array}{l}\text { Marital Status } \\
\text {-Married/De facto } \\
\text {-Divorced/Widowed/Separated } \\
\text {-Never married }\end{array}$ & $\begin{array}{r}51(23.7 \%) \\
31(14.4 \%) \\
133(61.9 \%)\end{array}$ & $\begin{array}{r}63(23.0 \%) \\
60(21.9 \%) \\
151(55.1 \%)\end{array}$ & $\begin{array}{r}114(23.3 \%) \\
91(18.6 \%) \\
284(58.1 \%) \\
489(100 \%)\end{array}$ & $0.101^{b}$ \\
\hline $\begin{array}{l}\text { Living arrangements } \\
\text { - With partner } \\
\text { - With parents/children (<18 years) } \\
\text { - With friends } \\
\text { - Alone } \\
\text { - Hostel/Shelter/Psych home/Homeless } \\
\text { - Other }\end{array}$ & $\begin{array}{r}48(22.3 \%) \\
73(34.0 \%) \\
21(9.8 \%) \\
50(23.3 \%) \\
13(6.0 \%) \\
10(4.7 \%)\end{array}$ & $\begin{array}{r}52(19.0 \%) \\
90(32.8 \%) \\
34(12.4 \%) \\
56(20.4 \%) \\
14(5.1 \%) \\
28(10.2 \%)\end{array}$ & $\begin{array}{r}100(20.4 \%) \\
163(33.3 \%) \\
55(11.2 \%) \\
106(21.7 \%) \\
27(5.5 \%) \\
38(7.8 \%) \\
489(100 \%)\end{array}$ & $0.229^{b}$ \\
\hline $\begin{array}{l}\text { Employment status } \\
\text { - Full time } \\
\text { - Part time/Student } \\
\text { - Retired/Disability/Home duties } \\
\text { - Unemployed }\end{array}$ & $\begin{array}{r}23(10.7 \%) \\
24(11.2 \%) \\
25(11.6 \%) \\
143(66.5 \%)\end{array}$ & $\begin{array}{r}33(12.0 \%) \\
22(8.0 \%) \\
27(9.9 \%) \\
192(70.1 \%)\end{array}$ & $\begin{array}{r}56(11.5 \%) \\
46(9.4 \%) \\
52(10.6 \%) \\
335(68.5 \%) \\
489(100.0 \%)\end{array}$ & $0.567^{\mathrm{b}}$ \\
\hline $\begin{array}{l}\text { Education Level } \\
\text { - Primary } \\
\text { - Partial Secondary } \\
\text { - Partial Secondary w/TAFE } \\
\text { - High School } \\
\text { - High School w/TAFE } \\
\text { - University/College }\end{array}$ & $\begin{array}{r}9(4.2 \%) \\
69(32.1 \%) \\
64(29.8 \%) \\
24(11.2 \%) \\
29(13.5 \%) \\
20(9.3 \%)\end{array}$ & $\begin{array}{r}4(1.5 \%) \\
89(32.5 \%) \\
91(33.2 \%) \\
25(9.1 \%) \\
37(13.5 \%) \\
28(10.2 \%)\end{array}$ & $\begin{array}{r}13(2.7 \%) \\
158(32.3 \%) \\
155(31.7 \%) \\
49(10.0 \%) \\
66(13.5 \%) \\
48(9.8 \%) \\
489(100 \%)\end{array}$ & $0.490^{\mathrm{b}}$ \\
\hline $\begin{array}{l}\text { Ethnic Background } \\
\text { - Caucasian } \\
\text { - Indigenous Australian } \\
\text { - Other } \\
\text { - Mixed background }\end{array}$ & $\begin{array}{r}164(80.4 \%) \\
14(6.9 \%) \\
5(2.5 \%) \\
21(10.3 \%)\end{array}$ & $\begin{array}{r}201(78.8 \%) \\
28(11.0 \%) \\
10(3.9 \%) \\
16(6.3 \%)\end{array}$ & $\begin{array}{r}365(79.5 \%) \\
42(9.2 \%) \\
15(3.3 \%) \\
37(8.1 \%) \\
459(93.9 \%)^{\star \star}\end{array}$ & $0.161^{b}$ \\
\hline
\end{tabular}

Abbreviation: TAFE, Technical and further education

${ }^{\mathrm{a}}$ Independent T-test, ${ }^{\mathrm{b}}$ Pearson chi-square test

**Total sums to less than $100 \%$ due to missing data and patients who refused to answer questions on ethnic background. 
RUNNING HEAD: ADHD and Substance Use Disorder Complexity

Table 2 - Association between ADHD status and SUDs reported stratified by specific drug type.

\begin{tabular}{|c|c|c|c|c|}
\hline $\begin{array}{l}\text { SUD } \\
N=489\end{array}$ & $\begin{array}{r}\text { ADHD-SP } \\
N(\%) \\
215(44.0 \%) \\
\end{array}$ & $\begin{array}{r}\text { ADHD-SN } \\
N(\%) \\
274(56.0 \%)\end{array}$ & $\begin{array}{r}\text { Crude OR } \\
(95 \% \mathrm{Cl})\end{array}$ & $\begin{array}{r}\text { Adjusted OR* } \\
(95 \% \mathrm{Cl})\end{array}$ \\
\hline \multicolumn{5}{|l|}{ Alcohol } \\
\hline Current & $121(56.3 \%)$ & $152(55.5 \%)$ & $1.03(0.72,1.48)$ & $1.16(0.75,1.79)$ \\
\hline History & $179(83.3 \%)$ & $206(75.2 \%)$ & $1.64(1.05,2.58)$ & $2.05(1.21,3.45)^{\star \star}$ \\
\hline \multicolumn{5}{|l|}{ Nicotine } \\
\hline Current & 99 (46.0\%) & $132(48.2 \%)$ & $0.92(0.64,1.31)$ & $0.93(0.61,1.42)$ \\
\hline History & $190(88.4 \%)$ & $243(88.7 \%)$ & $0.97(0.55,1.70)$ & $0.92(0.50,1.70)$ \\
\hline \multicolumn{5}{|l|}{ Heroin } \\
\hline Current & $66(30.7 \%)$ & 63 (23.0\%) & $1.48(0.99,2.22)$ & $1.36(0.84,2.21)$ \\
\hline History & $94(43.7 \%)$ & 109 (39.8\%) & $1.18(0.82,1.69)$ & $1.02(0.66,1.60)$ \\
\hline \multicolumn{5}{|l|}{ Methadone } \\
\hline Current & 45 (20.9\%) & 57 (20.8\%) & $1.01(0.65,1.56)$ & $0.96(0.57,1.63)$ \\
\hline History & $61(28.4 \%)$ & $80(29.2 \%)$ & $0.96(0.65,1.43)$ & $0.97(0.60,1.57)$ \\
\hline \multicolumn{5}{|c|}{ Other Opiates $^{a}$} \\
\hline Current & 45 (20.9\%) & 32 (11.7\%) & $2.00(1.22,3.28)$ & $1.56(0.89,2.74)$ \\
\hline History & $67(31.2 \%)$ & $57(20.8 \%)$ & $1.72(1.14,2.60)$ & $1.66(1.04,2.66)$ \\
\hline \multicolumn{5}{|c|}{ Benzodiazepines } \\
\hline Current & $55(25.6 \%)$ & $51(18.6 \%)$ & $1.50(0.98,2.32)$ & $1.51(0.91,2.50)$ \\
\hline History & 87 (40.5\%) & $80(29.2 \%)$ & $1.65(1.13,2.40)$ & $1.63(1.05,2.53)$ \\
\hline \multicolumn{5}{|l|}{ Cocaine } \\
\hline Current & $22(10.2 \%)$ & $18(6.6 \%)$ & $1.62(0.85,3.11)$ & $2.04(0.90,4.62)$ \\
\hline History & $44(20.5 \%)$ & $51(18.6 \%)$ & $1.13(0.72,1.76)$ & $1.10(0.65,1.88)$ \\
\hline \multicolumn{5}{|c|}{ Amphetamine } \\
\hline Current & 95 (44.2\%) & $76(27.7 \%)$ & $2.06(1.41,3.01)$ & $1.85(1.19,2.36)^{\star \star}$ \\
\hline History & $154(71.6 \%)$ & $141(51.5 \%)$ & $2.38(1.63,3.48)$ & $1.96(1.26,3.06)^{\star \star}$ \\
\hline \multicolumn{5}{|l|}{ Ecstasy } \\
\hline Current & $23(10.7 \%)$ & $14(5.1 \%)$ & $2.23(1.12,4.44)$ & $1.71(0.73,4.01)$ \\
\hline History & $68(31.6 \%)$ & $54(19.7 \%)$ & $1.89(1.25,2.85)$ & $1.62(1.00,2.61)$ \\
\hline \multicolumn{5}{|l|}{ Cannabis } \\
\hline Current & $94(43.7 \%)$ & 85 (31.0\%) & $1.73(1.19,2.51)$ & $1.58(1.00,2.51)$ \\
\hline History & 167 (77.7\%) & $200(73.0 \%)$ & $1.29(0.85,1.95)$ & $0.88(0.51,1.51)$ \\
\hline \multicolumn{5}{|c|}{ Other drugs $^{\mathrm{b}}$} \\
\hline Current & $5(2.3 \%)$ & $6(2.2 \%)$ & $1.06(0.32,3.53)$ & $0.79(0.22,2.86)$ \\
\hline History & $21(9.8 \%)$ & $25(9.1 \%)$ & $1.08(0.59,1.98)$ & $0.72(0.34,1.49)$ \\
\hline
\end{tabular}

${ }^{\star}$ model adjusted for age, gender, ethnicity, education level, living arrangements, marital status, offending history, and traumatic brain injury.

**p-value significant after Simes' method correction

a opiates other than heroin or methadone

bother drugs includes: LSD, barbiturates, ketamine, GHB, DMT, psilocybin mushrooms, Datura, synthetic cannabis, solvents, and sertraline. 
RUNNING HEAD: ADHD and Substance Use Disorder Complexity

Table 3 - Association between ADHD status and durations of SUD: Modified Poisson regression model analysis.

\begin{tabular}{|c|c|c|c|c|}
\hline $\begin{array}{l}\text { Duration of } \\
\text { Dependent Use }{ }^{a} \\
N=489\end{array}$ & $\begin{array}{r}\text { ADHD-SP } \\
N(\%) \\
215(44.0 \%) \\
\end{array}$ & $\begin{array}{r}\text { ADHD-SN } \\
N(\%) \\
274(56.0 \%) \\
\end{array}$ & $\mathrm{PRR}^{\star}(95 \% \mathrm{Cl})$ & $p$-value \\
\hline \multicolumn{5}{|l|}{ Alcohol $\geq 5$} \\
\hline Short (1-2 Years) & 17 (7.9\%) & $30(10.9 \%)$ & $1.01(0.61,1.67)$ & 0.982 \\
\hline Moderate (3-4 Years) & $21(9.8 \%)$ & $27(9.9 \%)$ & $1.39(0.92,2.10)$ & 0.123 \\
\hline Long ( $\geq 5$ Years) & $141(65.6 \%)$ & $149(54.4 \%)$ & $1.55(1.14,2.11)$ & $0.005^{\star \star}$ \\
\hline \multicolumn{5}{|l|}{ Other Opiates $^{b}$} \\
\hline Short (1-2 Years) & $34(15.8 \%)$ & 27 (9.9\%) & $1.30(1.00,1.69)$ & 0.049 \\
\hline Moderate (3-4 Years) & $8(3.7 \%)$ & $11(4.0 \%)$ & $0.80(0.42,1.52)$ & 0.500 \\
\hline Long ( $\geq 5$ Years) & $25(11.6 \%)$ & $19(6.9 \%)$ & $1.57(1.16,2.12)$ & $0.003^{\star \star}$ \\
\hline \multicolumn{5}{|l|}{ Benzodiazepines } \\
\hline Short (1-2 Years) & $22(10.2 \%)$ & $28(10.2 \%)$ & $1.00(0.70,1.43)$ & 0.984 \\
\hline Moderate (3-4 Years) & $22(10.2 \%)$ & $9(3.3 \%)$ & $1.61(1.22,2.13)$ & $0.001^{\star *}$ \\
\hline Long ( $\geq 5$ Years) & $43(20.0 \%)$ & $43(15.7 \%)$ & $1.31(1.01,1.68)$ & 0.039 \\
\hline \multicolumn{5}{|l|}{ Amphetamine } \\
\hline Short (1-2 Years) & $35(16.3 \%)$ & $38(13.9 \%)$ & $1.41(1.00,1.99)$ & 0.048 \\
\hline Moderate (3-4 Years) & $34(15.8 \%)$ & $22(8.0 \%)$ & $1.76(1.29,2.41)$ & $<0.001^{\star *}$ \\
\hline Long ( $\geq 5$ Years) & $85(39.5 \%)$ & $81(29.6 \%)$ & $1.46(1.11,1.93)$ & $0.008^{\star \star}$ \\
\hline \multicolumn{5}{|l|}{ Ecstasy } \\
\hline Short (1-2 Years) & $28(13.0 \%)$ & $18(6.6 \%)$ & $1.39(1.04,1.87)$ & 0.029 \\
\hline Moderate (3-4 Years) & $11(5.1 \%)$ & $15(5.5 \%)$ & $1.03(0.65,1.64)$ & 0.903 \\
\hline Long ( $\geq 5$ Years) & $29(13.5 \%)$ & $21(7.7 \%)$ & $1.30(0.97,1.73)$ & 0.081 \\
\hline
\end{tabular}

*model adjusted for age, gender, ethnicity, education level, living arrangements, marital status, offending history, and traumatic brain injury.

**p-value significant after Simes' method correction

a compared to a reference group of no years of dependent use

bopiates other than heroin or methadone

PRR: Prevalence Risk Ratio 